\title{
The Vibration Characteristics and Allowable Span Length of Free Span Submarine Pipeline
}

\author{
Bo ZHANG ${ }^{1}$, Ze-Yang XING ${ }^{1}$, Tao WANG $^{2}$ and Zhuo WANG ${ }^{1, a}$ \\ ${ }^{1}$ College of Mechanical and Electrical Engineering, Harbin Engineering University, Nantong Street \\ 145,150001 Harbin, China \\ 2 School of MechanicalEngineering, Hebei University of Technology, 300401 Tianjin, China
}

Keywords: Allowable span length, Free span, Submarine pipelines, Vibration characteristics.

\begin{abstract}
This paper focuses on the free span of submarine pipelines. The vibration differential equation and dynamic characteristic equation of submarine pipelines subjected to axial force and stress analysis were established, and then got the natural frequency. In order to avoid VIV, the vortex shedding frequency and reduced velocity was used as control parameters, and the allowable span length of submarine pipelines was thus derived. By using calculation example, to analyze the influence of flow velocity, axial force, and pipe dimensions on the natural frequency and allowable span length. The general method for determining was given. The result indicates that the allowable span length of submarine pipelines decreases with flow velocity, compression, and increases with an increment in tension.
\end{abstract}

\section{Introduction}

Subsea pipelines are an important part of the development system of marine oil and gas[1], which is transmission of continuous, easy management, high efficiency, low cost and other advantages, known as the of offshore oil and gas fields. However, submarine topography and geological structure, and the scouring action of wave and current, submarine sand waves or dune movement and the change of soil properties, etc, can cause the occurrence of submarine pipeline free span defined as the impending period which not direct contacted between the surface of the submarine pipeline and seabed[2]. The emergence of the span changes the load borne by the state and stress of the submarine pipelines, the vortex shedding is often accompanied, when the water flows through the pipelines. That phenomenon of vortex shedding will cause the in-line or cross-flow motion of pipelines that are vortex-induced vibration [3]. Vortex induced vibration (VIV) is the key to deciding to service life of a submarine pipeline and lead to the destruction of pipelines. When the natural frequency is near to the vortex shedding frequency, it can lead to spinning vortex-induced resonance of pipelines. Therefore, the study of vibration characteristics of span submarine pipelines has important significance to ensure the safe operation of submarine pipelines in service period.

In the aspect of submarine spanning pipeline, the scholars both at home and abroad have done a lot of research work, and also proposed many methods of the calculation of allowable span length and evaluation of pipeline vortex-induced vibration. This paper focuses on the free span of submarine pipelines. According to the theory of Euler-Bernoulli beam, the vibration control equation of spanning pipe was deduced. By using two different control parameters to find the allowable span length of spanning pipelines, and analyze the influence of factors on the natural frequency and allowable span length.

\section{The Mathematical Model of Free Spanning Pipeline}

Free spanning pipeline can be seen as a uniform thin beam of cross section, and assuming transverse displacement $\mathrm{y}(\mathrm{x}, \mathrm{t})$ is caused only by bending. The bending vibration is done in symmetric in-plane itself. The model is based on elementary beam theory of Euler-Bernoulli beam[4]. Among the vibration system, the main physical properties are flexural rigidity $\mathrm{EI}(\mathrm{x})$ and the mass of pipe per unit length $\mathrm{m}(\mathrm{x})$, both of them change along the location $\mathrm{x}$. The boundary 
condition of pipe in Figure. 1 is one ends hinged and the other ends fixed. But the motion equation deduced suit arbitrarily conditions.

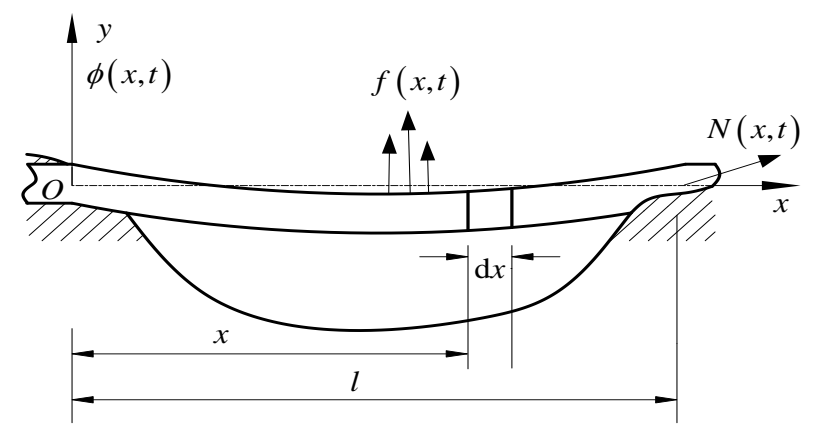

Figure.1 Free spans of submarine pipeline

In the figure, the span length is 1 , axial tension is $\mathrm{N}(\mathrm{x}, \mathrm{t})$, and the external force of pipe of per unit length is $f(x, t)$.

\section{The Vibration Equation of Pipelines and Solution}

The process of pipe element is in Figure.2. In the figure, element $\mathrm{dx}$ is taken from the displace $\mathrm{x}$ of pipe. Where, $\mathrm{M}$ is bending moment, $\mathrm{Q}$ is shearing force, and assuming the axial force is constant, that unchanged long time and location.

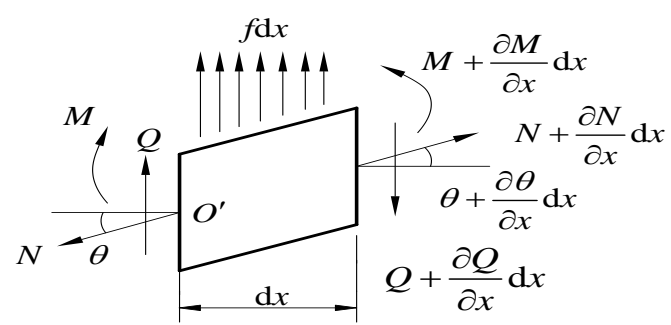

Figure.2 Process of pipe element

In Figure.2, for the vertical vibration, the motion equation of the pipe element can be obtained

$$
\begin{aligned}
& -(Q+\mathrm{d} Q)+f \mathrm{~d} x+Q+(N+\mathrm{d} N) \sin (\theta+\mathrm{d} \theta) \\
& -N \sin \theta=\rho A \mathrm{~d} x \frac{\partial \phi}{\partial t}
\end{aligned}
$$

For the rotation relative to $O^{\prime}$, there be

$$
(M+\mathrm{d} M)-(Q+\mathrm{d} Q) \mathrm{d} x+f \mathrm{~d} x \frac{\mathrm{d} x}{2}-M=0
$$

For small deformation, approximate may then be expressed by

$$
\sin (\theta+\mathrm{d} \theta) \approx \theta+\mathrm{d} \theta=\theta+\frac{\partial \theta}{\partial x} \mathrm{~d} x
$$

According to Eq. (1), (2), (3), and Material mechanics formula are given by

$$
M=E I(x) \frac{\partial^{2} y}{\partial x^{2}}
$$

The differential equation of motion can be derived, where $E I, m$ and $N$ is constant, the equation is 


$$
E I \frac{\partial^{4} \phi}{\partial x^{4}}+m \frac{\partial^{2} \phi}{\partial t^{2}}-N \frac{\partial^{2} \phi}{\partial x^{2}}=0
$$

The solution of Eq. (5) can be solved by separation of variable

$$
\begin{aligned}
\Phi(x)= & C_{1} \cosh s_{1} x+C_{2} \sinh s_{1} x+C_{3} \cos s_{2} x \\
& +C_{4} \sin s_{2} x
\end{aligned}
$$

Where

$$
s_{1}^{2}, s_{2}^{2}=\frac{N}{2 E I} \pm\left(\frac{N^{2}}{4 E^{2} I^{2}}+\frac{m \omega^{2}}{E I}\right)^{1 / 2}
$$

The constant $C_{1}, C_{2}$ can be determined on the basis of boundary conditions.

\section{The Solution of Natural Frequency is Under the Condition of Both Ends Hinged}

The purpose of this part is to get the natural frequency of lateral vibration. The model the boundary condition is

$$
\phi(0)=0, E I \frac{d^{2} \phi(l)}{d x^{2}}=0 ; \phi(l)=0 E I \frac{d^{2} \phi(l)}{d x^{2}}=0
$$

When substitute Eq. (8) into Eq. (6), the natural frequency under the condition of both ends hinged can be got

$$
\omega_{n}=\frac{\pi^{2}}{l^{2}} \sqrt{\frac{E I}{m}}\left(n^{4}+\frac{n^{2} N l^{2}}{\pi^{2} E I}\right)^{1 / 2}
$$

Where axial force is pressure, $N$ is negative, axial force is tension, $N$ is positive.

\section{The Natural Frequency of Free Spanning Pipeline under General Conditions}

The natural frequency of free spanning pipeline can be obtained in the same way before. One order natural frequency is found under three kinds of conditions, then simplifies to

$$
f_{n}=\frac{C}{l^{2}} \sqrt{\frac{E I}{M}}
$$

Where $C$ is the constant related to boundary conditions of free spanning pipeline [5]. When the condition is both ends hinged, the number of $C$ gets 1.57, the $C$ is 3.56 with the condition of both ends fixed, and the $C$ is 2.46 between the conditions before. $M$ is mass of pipe per unit length (that includes medium quality in the pipe and the added mass of water). Unit is $\mathrm{kg}$.

In most cases, the boundary condition of free spanning pipeline is not both ends hinged. Neither both ends fixed, factors of conditions include Soil stiffness and surroundings around the pipe. In general, the condition is often taken between them [6].

Eq.(9) and Eq.(10) show that: (1) if $\mathrm{N}=0$, the natural frequency found from Eq.(9) and Eq.(10) under the condition of both ends hinged are similar; (2) if $N>0$ (axial tension), the natural frequency increases with tension increases;(3) if $N<0$ (pressure), the natural frequency decreases with pressure increases; if $N \rightarrow \pi^{2} E I / l^{2}$, for

$$
f_{D}^{\prime}=\frac{1}{2} C_{D}^{\prime} \rho D U^{2} \cos \left(4 \pi f_{s} t\right)
$$

The natural frequency is near to zero. 


\section{Control Condition and Allowable Span Length}

When the fluid is close to the front of the pipeline, the pressure of fluid will be increases because of the resistance. The increased pressure distributes around the boundary layer of the pipeline surface along the downstream on both sides, and separates near the point, which has the largest width of the pipeline cross section. The point speeds from positive to negative or zero velocity point is called as the separation point. After the separation point, the backflow will occur along the pipeline surface. The boundary layer is out of the pipeline surface on the separation point and become the free shear layer, which extends along the downstream. Between the both sides of the shear layer is the wake zone. Within the shear layer, the velocity of the lateral part, which is close to the free flow area, is greater than the internal one, so the fluid will rotate and disperse into many vortexes. Vortexes occur periodically and alternately on the left and right sides of the pipeline and lead to the vibration of the pipeline. When the frequency of vortexes breaking is close to the natural frequency of the pipeline, they will change together. So energy is input into the pipeline by the wake resonant with the large amplitude vibration. In this case, it is easy for the pipeline to be destroyed. So in engineering, the allowable free span length of the submarine pipelines should be calculated. Submarine pipelines hanging over length should be repaired [7].

One of the criteria to avoid VIV is that the vortex shedding frequency less than 0.7 times of natural frequency. The vortex shedding frequency, $f_{\mathrm{s}}, f_{s}=S_{t} U / D$. The other criterion is the reduced velocity, $V_{r}=U /\left(f_{n} D\right)$. According to Document [8], when $4.5<V_{r}<10$, the pipe is easy to occur lock phenomenon, this paper takes 4.5 of the control condition to avoid cross-flow VIV of free spanning pipeline. Equations of allowable span length are given by

$$
\begin{aligned}
& l \leq \sqrt{\frac{0.7 C D}{S_{t} U} \sqrt{\frac{E I}{M}}} \\
& l \leq \sqrt{\frac{C D V_{r \max }}{U} \sqrt{\frac{E I}{M}}}
\end{aligned}
$$

The Strouhal number, $S_{\mathrm{t}}$, is a non-dimensional number which relates the vortex shedding frequency, the diameter of the cylindrical pipe, and the velocity of the flow. For submarine pipeline, $S_{\mathrm{t}}$ is taken as 0.2 to calculate. The mass of pipe per unit length $\mathrm{M}$ is given by

$$
M=\frac{\pi}{4}\left[\left(D_{o}^{2}-D_{i}^{2}\right) \rho_{s}+\left(D_{c}^{2}-D_{o}^{2}\right)+D_{i}^{2} \rho_{f}+D_{c}^{2} \rho_{w}\right]
$$

\section{Calculation and Analysis on an Example}

The model of the submarine is monolayer steel pipeline with weight coating of concrete. The weight coating of concrete only considers quality and ignores the influence of the stiffness. The pipe is buried in the seabed with a period of free span in the middle scored by current. The model is in Figure.3. The Parameters of the pipe is in Table 1.
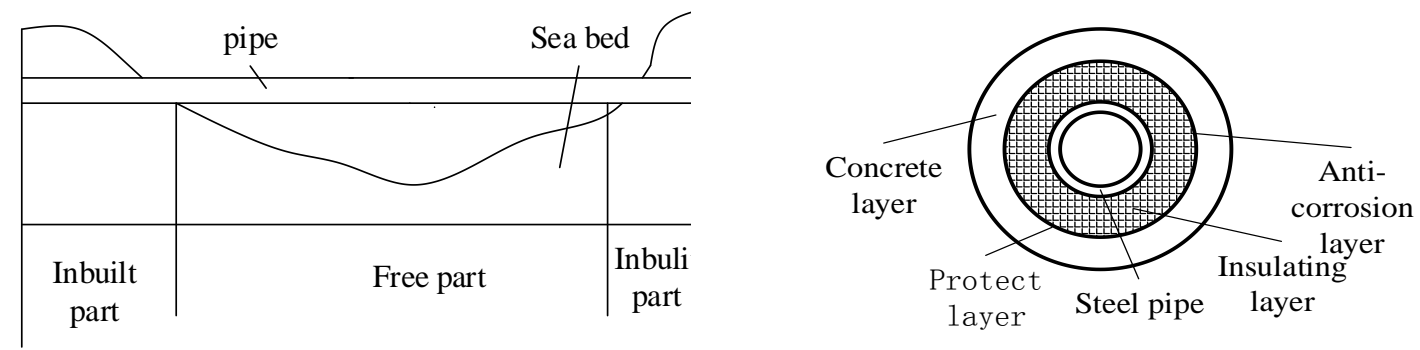

Figure.3 Model of submarine pipeline 
Table 1. Parameters of the pipe

\begin{tabular}{|c|c|c|}
\hline Parameters & Value & Implication \\
\hline$D o / \mathrm{m}$ & 0.5588 & Steel pipe diameter \\
\hline$D i / \mathrm{m}$ & 0.527 & Steel pipe ID \\
\hline$D c / \mathrm{m}$ & 0.68 & Concrete layer OD \\
\hline$t c / \mathrm{mm}$ & 60 & Concrete thickness \\
\hline$\rho_{c} / \mathrm{kg} \cdot \mathrm{m}^{-3}$ & 3040 & Concrete layer density \\
\hline$\rho_{s} / \mathrm{kg} \cdot \mathrm{m}^{-3}$ & 7850 & Density of steel pipe \\
\hline$\rho_{f} / \mathrm{kg} \cdot \mathrm{m}^{-3}$ & 908.2 & Oil density \\
\hline$\rho_{w} / \mathrm{kg} \cdot \mathrm{m}^{-3}$ & 1025 & Seawater density \\
\hline$E / \mathrm{Pa}$ & $2.07 \mathrm{E} 10^{11}$ & Young modulus \\
\hline
\end{tabular}

From the formula above, the factors that affect natural frequency and allowable span length include boundary conditions, axial force, mass of pipe per unit length, etc. Among them, the influence of mass of pipe per unit length is complicated on natural frequency and allowable span length.

\section{Effect of Boundary Conditions on Natural Frequency}

Figure. 4 shows the relationship between natural frequencies and span lengths under different boundary conditions.

In the figure, with the increase in pipeline span length, the natural frequency of the pipeline gradually reduces under three kinds of boundary conditions. And in the same span length, the natural frequency is minimal with the condition of both ends hinged. In this condition, the pipe is easy to lead to vortex-induced resonance.

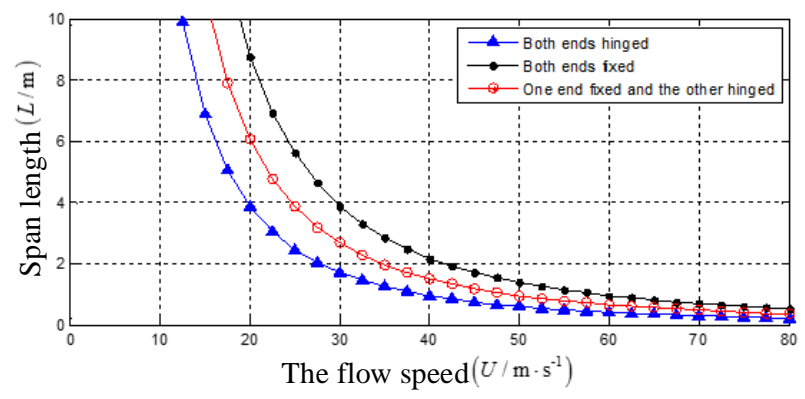

Figure. 4 The relationship between pipe natural frequencies and span lengths under different boundary conditions

\section{Effect of the Tube Medium on Natural Frequency}

The submarine pipeline is used to transport oil and gas, the situation is different whether is being fluid or not. Figure. 5 shows the relationship between pipe natural frequencies and span lengths under different liquid. In the Figure.5, in the same span length, the natural frequency of pipe with fluid is lower than that with no fluid. Therefore, the pipeline using and the server is easy to bring up vortex-induced resonance. Meanwhile, with the increase of span length, the natural frequency decreases. Figure. 5(a) is in the condition of both ends hinged and Figure. 5(b) is in the condition of both ends fixed. 


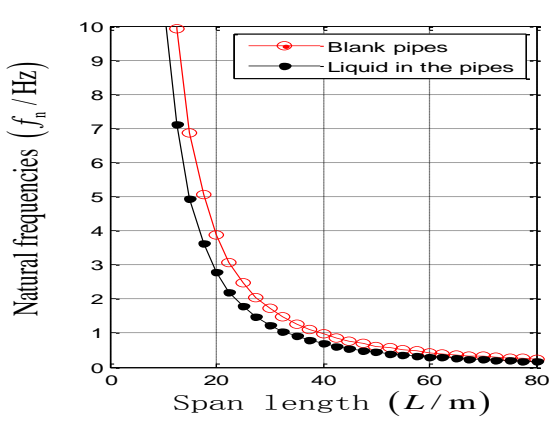

(a)

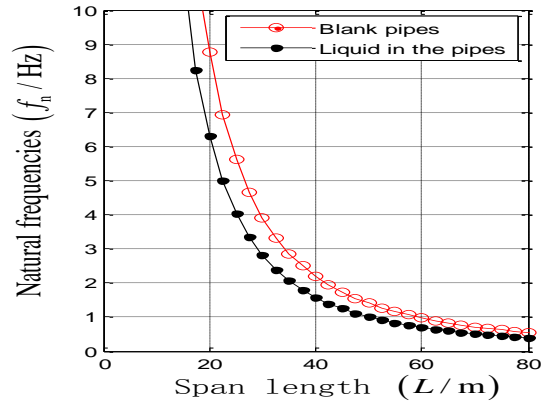

(b)

Figure.5 The relationship between pipe natural frequencies and span lengths under different liquid

\section{Effect of Flow Velocity on Span Length}

Figure. 6 shows the effect of the flow speed on pipe span length under different boundary conditions.

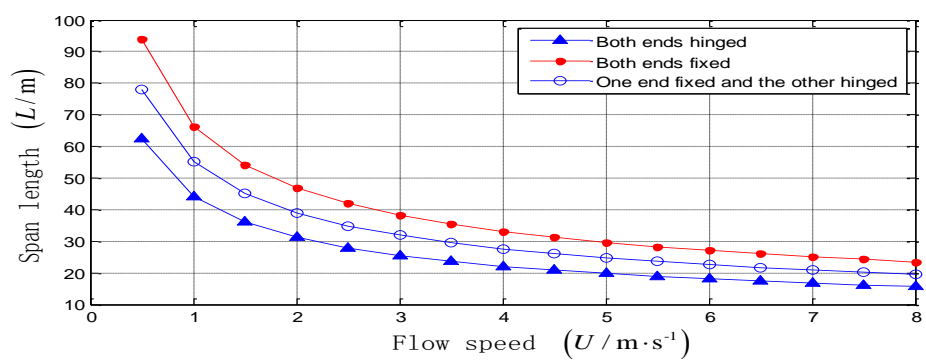

Figure.6 effect of the flow speed on pipe span length under different boundary conditions

In the figure, with the increase of current velocity, the allowable span length decreases. For the same flow velocity, the allowable span length is not the same under different support conditions.

\section{Effect of the Axial Force on Span Length}

Supposing the flow speeds $U=1.2 \mathrm{~m} / \mathrm{s}$ under the condition of both ends hinged. The internal flow velocity and pressure are not considered. Changing axial force and the corresponding allowable span length is obtained in Figure.7. In the figure, tensile stress can increase the allowable span length of pipe, with the increase of tensile stress, the allowable span length increases as well; Compressive stress will make allowable span length of pipe decrease, with the increase of compressive stress, the allowable span length will be reduced accordingly. Therefore, there should reduce the occurrence of compressive stress as far as possible during the laying and use of pipe.

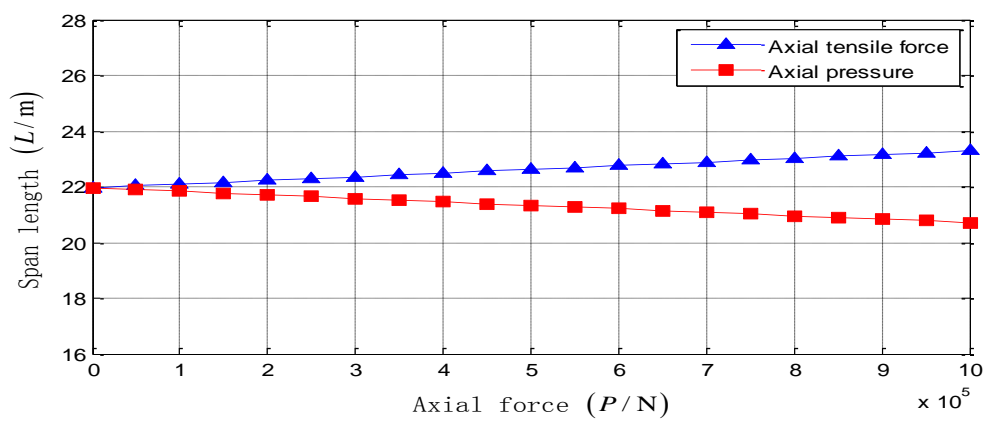

Figure.7 Effect of the axial force on span length

\section{Effect of Pipe Size on Span Length}

Fig. 8 shows the effect of pipe size on span length, the span length increases with the pipe diameter increases. As different specifications of pipe diameter, its weight, the added mass and the quality of the medium is not the same. And that will influent the buoyancy of pipe, lift force, the vortex 
shedding frequency, etc. So the influent of the pipe size on the natural frequency and allowable span length is complicated.



Figure.8 Effect of the pipe size on span length

There are a lot of factors that affect the natural frequency and allowable span length. In addition to the above, there are other factors. Such as the internal flow velocity, temperature, pressure, damp, etc. Studies have shown that the axial force to the allowable span length is larger than the influence of other factors [9].

\section{Determination of Allowable Span Length}

The results of two calculation methods Eq. (11) and Eq. (12) to the allowable span length are similar. The two methods for allowable span length under the condition of both ends hinged are presented in Figure.9. For the same flow rate $U=1.2 \mathrm{~m} / \mathrm{s}$, as well as the parameters agreed in two formulas, results of span length are similar, as showed in Table 2.

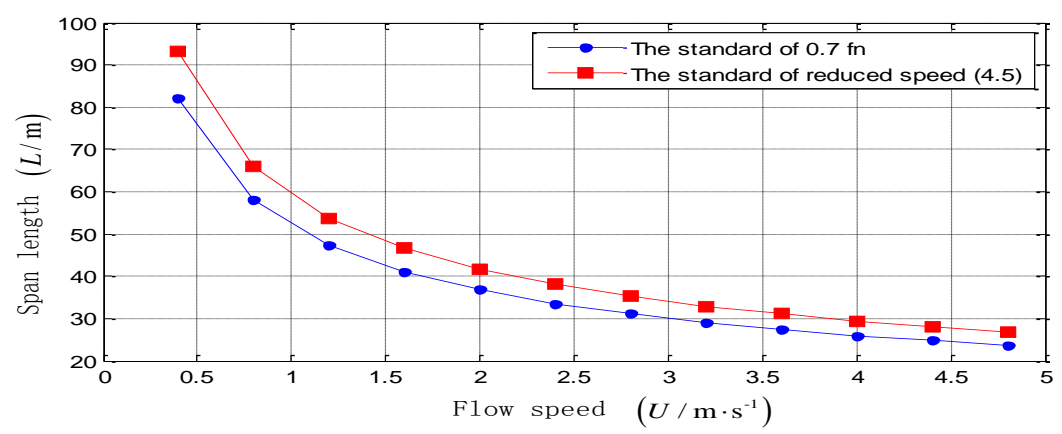

Figure. 9 the two methods for allowable span length

Table 2. Calculation of allowable span length

\begin{tabular}{|c|c|c|c|}
\hline Control parameter & $f_{s} \leq 0.7 f_{n}$ & $V_{r}<V_{r_{\max }}$ & Boundary conditions \\
\hline \multirow{3}{*}{ Allowable span length /m } & 36.4 & 41.3 & Both ends hinged \\
\cline { 2 - 4 } & 54.8 & 62.1 & Both ends fixed \\
\cline { 2 - 4 } & 45.5 & 51.4 & Between the tows \\
\hline
\end{tabular}

According to the analysis before, the pipeline dimensions, boundary conditions and the outside wave and current conditions all have an influence on the maximum spanning length. Thus, there will not be a specific date and it depends on specific circumstances. Theoretically, the allowable spanning length is the min result among the results from different kinds of methods. But in fact, the allowable spanning length should be obtained by comparing all the results to get a reasonable data. And special considerations should be taken under specific circumstances. If the spanning appears in 
the very deep seabed, that velocity of flow is almost zero; using static strength method is enough. If it requires high level of security, using the min of movement method to assess is needed.

\section{Conclusions}

The free spanning pipeline was simplified to the Euler-Bernoulli beam. Vibration control equations were deduced and solving the natural frequency under different boundary conditions. The vortex shedding frequency and reduced velocity was used as control parameters to derive the allowable span length of submarine pipelines. Therefore, the influence of flow velocity, axial force, and pipe dimensions on the natural frequency and allowable span length was discussed. Through the example analysis can draw the following conclusions:

(1) The flow velocity, axial force, boundary conditions and as well as pipe dimensions have an effect on the natural frequency of pipelines. The first order natural frequency corresponded to the main vibration among the natural frequencies derived from the theory is the smallest. The factors or trends of them make the natural frequency decrease, and then free spanning pipelines get easy to occur vortex induced resonance under this condition. Conversely, that can avoid the vortex-induced resonance.

(2) The influence of the axial force on allowable span length of pipelines should not be neglected during laying and servicing of submarine pipelines. Tensile stress can increase the allowable span length of pipe, with the increase of tensile stress, the allowable span length increases as well; Compressive stress will make allowable span length of pipe decrease, with the increase of compressive stress, the allowable span length will be reduced accordingly. Therefore, there should reduce the occurrence of compressive stress as far as possible during the laying and use of pipe.

(3) In engineering applications, the calculation of sea pipeline free span length shall be calculated according to the actual situation and then choose a calculation formula for the suspended span length.

\section{Acknowledgements:}

This material is based upon work supported by the National Science Foundation of China (Grant no. 51479043). The views expressed are authors alone.

\section{References}

1. D.Y.Zhao, J.X.Yu, G.X.Li etal. J Harbin Eng Univ,6,597-601(2009)

2. L.Li, Z.D.Zhang . Ship Build CHN,191,13-17(2010)

3. G.X.Wang. Qingdao: Ocean University of China, 34-36 (2006)

4. V.Knut, S.Havar, H.Jostein. Eng Strcut, 56, 68-82(2013)

5. H.H,Shao, H.Q.Hu, Z.F.Xu.. Pet Plan Des, 6,27-2(2003)

6. H.S. Choi. Ocean Eng, 28, 1325-1338(2001)

7. J.X.Yu, Y.J.Ma, Y.Yang etal. J Tianjin U Tech.,1, 6-10 (2014)

8. G.C.Wang. Tianjin University,53-58 (1987)

9. X.H.Yang, H.Y.Guo, M.Lou etal. Ocean Eng, 1,1-5(2005)

10. Luigi T.De Luca, Propulsion physics (EDP Sciences, Les Ulis, 2009)

11. F. De Lillo, F. Cecconi, G. Lacorata, A. Vulpiani, EPL, 84 (2008) 